Rev. Int. Contam. Ambie. 37, 357-371, 2021

https://doi.org/10.20937/RICA.53758

\title{
CLASIFICACIÓN Y AFECTACIÓN POR RESIDUOS SÓLIDOS URBANOS EN LA CIUDAD DE LA PLATA, BUENOS AIRES, ARGENTINA
}

Classification and effects of urban solid waste in the city of La Plata, Buenos Aires, Argentina

\author{
Jesica ESPARZA
}

Instituto de Investigaciones y Políticas del Ambiente Construido, Facultad de Arquitectura y Urbanismo, Universidad Nacional de La Plata, CONICET.

Correo electrónico: esparzajesicabelen@gmail.com

(Recibido: octubre de 2019 aceptado: junio de 2020)

Palabras clave: contaminación, basurales urbanos, análisis y ubicación de basurales, población afectada.

\section{RESUMEN}

La generación de residuos sólidos urbanos representa una de las mayores problemáticas de la actualidad. En primer lugar, porque el volumen y la composición de la basura se ha incrementado y modificado por el nuevo estilo de consumo (se redujo el desecho orgánico, de papel y vidrio y aumentó el de plástico) y, en segundo lugar, por ser una problemática social, producto de la situación económica de los modelos neoliberales actuales. Este trabajo fue realizado en la ciudad de La Plata, Buenos Aires, Argentina, en donde se han analizado basurales de difícil erradicación (basurales de gran escala) y de fácil erradicación (basurales de escala media y pequeña). Como resultado se determinó que la población afectada es de 46010 habitantes, de los cuales el $46 \%$ pertenece a basurales en áreas inundables y el $32.45 \%$ a basurales en asentamientos precarios. En el caso de los basurales de difícil erradicación, aquellos que comprometen imperiosamente la salud pública, se observó que los mismos promueven la proliferación de vectores contaminantes -acuíferos y terrestres, así como enfermedades cutáneas y respiratorias en niños y adultos mayores. El origen de los mismos, generalmente se produce por la falla en el sistema de infraestructura integral y luego, por ser vertederos ilegales de uso público y privado. En tal sentido, la identificación y caracterización de los mismos, así como la cantidad de población y superficie afectada, permitiría determinar acciones para una eventual mitigación y/o erradicación como parte de un plan de ordenamiento urbano integral.

Key words: contamination, urban garbage dumps, garbage dumps analysis and location, affected population.

\begin{abstract}
The generation of urban solid waste is one of the mayor problems today. Firstly, because the volume and composition of waste has been increased and modified by the new style of consumption (organic, paper and glass waste reduced and plastic waste increased) and secondly, because it is a social problem, a product of the economic situation of current neoliberal models. This paper was carried out in the city of La Plata, Buenos Aires, Argentina, where landfills of difficult eradication (large scale landfills) and easy eradication (medium and small scale landfills) have been analyzed. As a result,
\end{abstract}


it was determined that the affected population is 46010 inhabitants, of which 46 per cent belong to landfills in floodable areas and 32.45 per cent to landfills in precarious settlements. In the case of landfills that are difficult to eradicate, those that imperiously compromise public health, it was observed that they promote the proliferation of polluting vectors - aquifers and terrestrial, as well as skin and respiratory diseases in children and older adults. The origin thereof, usually caused by system failure comprehensive infrastructure and then, being illegal landfills of public and private use. In this sense, their identification and characterization, as well as the quantity of population and affected area, would allow determining actions for eventual mitigation and / or eradication as part of a comprehensive urban planning plan.

\section{INTRODUCCIÓN}

La gestión de los residuos sólidos urbanos (RSU) se ha convertido en una problemática en ascenso, principalmente por los procesos de urbanización de las ciudades, ya que existe una clara desarticulación entre la planificación urbana - muchas veces ineficiente o nula - y la eliminación de basura. A partir de la segunda mitad del siglo 20, las urbanizaciones han crecido exponencialmente, situándose en sectores no aptos para asentamientos humanos, sin correspondencia entre usos e infraestructura de servicios básicos (D'hers 2013).

Los RSU se han incrementado a nivel global como resultado del aumento de población, cambios en el estilo de vida, perfiles de consumo, edad de integrantes de las familias, así como tendencias industriales y comerciales que promueven el consumo de una mayor cantidad de productos manufacturados (OECD 2004).

Alrededor de una tercera parte de los residuos generados en América Latina y el Caribe (145000 t/día) terminan en basureros a cielo abierto, son quemados o eliminados de otras formas inadecuadas. El programa de la ONU para el medio ambiente impulsó una coalición voluntaria que incluye lineamientos para el cierre progresivo de los basurales, la elaboración de guías técnicas y acciones de capacitación e intercambio de información (ONU 2019). ${ }^{1}$

Por otro lado, según el último "Informe del estado del ambiente" publicado en 2018 por la entonces Secretaría de Gobierno de Ambiente y Desarrollo Sustentable de Argentina (SGAyDS 2018), ${ }^{2}$ un estudio que se realizó en 164 países dejó en evidencia que la generación de basura a nivel mundial asciende a 1900 millones de toneladas anuales. En Argentina, el manejo de los RSU se encuentra regulado por la Ley N. ${ }^{\circ} 25916$ de Gestión de Residuos Domiciliarios, la cual los define como "aquellos elementos generados por procesos de consumo en el ámbito residencial, urbano, comercial, sanitario o industrial, que luego de su uso son desechados y/o abandonados", la cual establece presupuestos mínimos de protección ambiental para su tratamiento integral, así como un marco regulatorio y operativo que distingue diferentes categorías y su disposición final (SGAyDS 2018).

Entre las problemáticas derivadas de los basurales sin gestión se encuentran aquellas ligadas a la salud pública. Es una problemática en ascenso, ya que no sólo provocan la proliferación de vectores contaminantes, sino que representan un riesgo potencial de contagio para adultos mayores y grupos vulnerables, así como mayor susceptibilidad a enfermedades dérmicas y respiratorias de la población lindante y recuperadores urbanos. En la mayoría de los casos los afectados se contagian a través del contacto con animales domésticos, roedores infectados o por la misma ingesta de alimentos descompuestos que se encuentran en dichos basurales (ISWA 2015). Por otro lado, la disposición de residuos a cielo abierto ${ }^{3}$ provoca un importante deterioro ambiental de las aguas subterráneas por infiltración de lixiviados; de las aguas superficiales por escorrentía del suelo mediante filtración de lixiviados - microorganismos patógenos, metales pesados, sales e hidrocarburos clorados-, y del aire como producto de la quema

1 Hasta el momento participan en la iniciativa los gobiernos de Argentina, Cuba, Ecuador, El Salvador, Guatemala, Honduras, Panamá, República Dominicana, Surinam y Uruguay.

2 A partir de 2019 la Secretaría de Gobierno de Ambiente y Desarrollo Sustentable de Argentina se convirtió en Ministerio de Ambiente y Desarrollo Sustentable de la Nación Argentina.

3 Basural a cielo abierto: lugar de disposición final de los residuos sólidos urbanos y peligrosos, generalmente sin control de operación. 
—ausencia del control de humo y gases-(SGAyDS 2018).

En el caso de la ciudad de La Plata, como muchas otras ciudades del conurbano bonaerense, uno de los factores que ha incentivado el incremento de dichos residuos es el crecimiento y concentración de la población en áreas urbanas y suburbanas carentes de infraestructura óptima para su recolección. Las opciones de tratamiento y disposición final de residuos en el Partido de La Plata están limitadas por el marco legal vigente, el Decreto Ley N. ${ }^{\circ}$ 9111/78, el cual establece la obligatoriedad para la Ciudad Autónoma y 22 Partidos del Gran Buenos Aires de disponer los residuos en la Coordinación Ecológica Área Metropolitana Sociedad del Estado (CEAMSE), mediante el sistema de relleno sanitario, en predios y con las tarifas que éste determine. De acuerdo con el CEAMSE, que recibe aproximadamente el $40 \%$ de los residuos del país, en 2018 procesó siete millones de toneladas de RSU con una clara preponderancia de material orgánico, seguido por plásticos, papel y cartón.

En estos momentos, la Municipalidad de La Plata consta de un programa que comprende la gestión de la recolección diferenciada por tipo de residuos, la profesionalización del personal y la concientización para beneficiar el cuidado del medio ambiente y lograr una ciudad sustentable, ${ }^{4}$ sin embargo, quedan muchas áreas - principalmente del periurbano - sin cubrir. Por lo tanto, el principal conflicto ambiental de la región en cuanto a la gestión de los residuos urbanos es la creciente acumulación transitoria o crónica de residuos en la modalidad de montículos y pequeños, medianos o grandes basurales. Como se ha mencionado, La Plata no escapa a la tendencia general sobre la composición de los basurales, pasando de ser densa y casi completamente orgánica, con gran predominio de biodegradables, a ser voluminosa, menos biodegradable y con porcentajes crecientes de materiales peligrosos (D’hers 2013). En tal sentido, cabe mencionar que no hay al momento informe alguno que especifique la cantidad, ubicación, composición, volumen o cualquier otra característica de los basurales de la ciudad de La Plata; sin embargo, entre 2017 y 2019 el concejal platense Gastón Crespo (2017) impulsó un proyecto en que la ciudadanía podía dejar sus reclamos respecto a esta situación. ${ }^{5}$
Se registraron más de 126 denuncias, distribuidas en toda la región, que arrojaron lo siguiente: 1) la mayor cantidad de denuncias se registraron en primer lugar en la delegación de San Carlos (26 denuncias) y, en segundo lugar, en la delegación de Villa Elvira (23 denuncias); 2) 42 de dichos basurales se asientan sobre áreas con riesgo hídrico; 3) 20 se encuentran en torno a asentamientos precarios; 4) 67 son de carácter puntual, es decir, que se encuentran en una esquina o son de volumen reservado; 5) 91 tienen su origen en la disposición y vuelcos de carácter público y privado; 6) 93 son de carácter constante en el tiempo, es decir, perduran en cuanto a su conformación, aunque su volumen sea constante, y 7) todos son de composición mayoritariamente orgánica.

En consecuencia, se plantea esta investigación, en la cual se identifican y clasifican los basurales de la ciudad de La Plata, así como el grado de afectación a la población circundante. Adicionalmente, los resultados aquí descritos se presentan como insumo para mejorar o implementar futuros programas para la gestión y tratamiento de RSU en el municipio.

\section{MATERIALES Y MÉTODOS}

\section{Descripción del área de estudio}

El Partido de La Plata se ubica en el noreste de la provincia de Buenos Aires, situado a $60 \mathrm{~km}$ de la Ciudad Autónoma de Buenos Aires. Ocupa una superficie de $821 \mathrm{~km}^{2}$, con una altura de 0 a $20 \mathrm{~m}$ sobre el nivel del mar. Se caracteriza por la situación geomorfológica de llanura (pampeana) y su clima es templado húmedo (Hurtado et al. 2006). Consta de un casco urbano de consolidación alta-media, el cual se ha ido extendiendo hacia la periferia, de consolidación media-baja hacia Tolosa, Ringuelet, Manuel B. Gonnet, City Bell, Villa Elisa, Melchor Romero, Abasto, Gorina, José Hernández, Ángel Etcheverry, Arturo Seguí, Los Hornos, Lisandro Olmos, Villa Elvira y Altos de San Lorenzo, todos ellos con centros comunitarios que funcionan como delegaciones locales. Al mismo tiempo, se encuentra atravesada por una serie de arroyos, cuya vertiente desagua en la planicie costera de Berisso y Ensenada, generando una serie de bañados naturales (Karol y San Juan 2018); sin embargo, esta situación enmarca un contexto de

4 La recolección de residuos en la ciudad de La Plata se divide en residuos secos, húmedos y no habituales.

5 Portal ciudadano donde se elige una categoría y se completa un formulario para realizar un reclamo sobre residuos en la vía pública. Proyecto impulsado por el Concejal Gastón Crespo, La Plata, Buenos Aires, Argentina, 2019. Acceso libre y gratuito. 
fragilidad hídrica, provocando un estado de riesgo cada precipitación severa. En la figura 1 se observan la ciudad de La Plata, las delegaciones municipales y las cuencas de los arroyos que la atraviesan.

En la década de 1990 las nuevas formas urbanas en Latinoamérica dieron lugar a la denominada "ciudad fragmentada", de carácter marcadamente insular, segregativa y divisoria. En este marco, los procesos de transformación socioterritoriales que acontecieron en el ámbito urbano latinoamericano dieron lugar a territorios caracterizados por fenómenos tales como la dispersión, la fragmentación, la segregación, la exclusión y la informalidad urbana. En tal sentido, el crecimiento de la ciudad de La Plata se ha dado principalmente por extensión y consolidación, sin regulación ni legislación municipal. Por lo tanto, los nuevos asentamientos - tanto formales como informales - se encuentran desprovistos de infraestructuras aptas para un correcto desarrollo de la habitabilidad. Producto de este panorama, la población con menores recursos económicos constituye el grupo de mayor vulnerabilidad socioterritorial, asentada principalmente en sectores marginados. Específicamente, los basurales en estos sectores se originan principalmente por dos cuestiones: en primer lugar, por el tipo de actividad laboral que se realiza, la cual se basa fundamentalmente en changas, cartoneo o cirujeo (que es la recolección de cartón y otros derivados del papel por las calles de las ciudades), lo que constituye el origen de los basurales en torno a las viviendas, acompañado por la falta de presencia estatal en relación con la gestión integral de residuos; en segundo término, por la presencia de tierras vacantes, propias del tipo de ocupación del suelo actual, la cual se encuentra regida principalmente por el precio de la tierra. Dichos vacíos intersticiales del tejido urbano promueven la aparición, proliferación y expansión de basurales en áreas periféricas (Discoli et al. 2013).

Siguiendo con lo anterior, estos basurales nunca salen del circuito de valorización ya que, en primera instancia, son residuos que debe gestionar el propio Estado (recolección y transporte) hasta su disposición final en un relleno sanitario por la vía legal, pero que finalmente terminan siendo repositorios ilegales de los habitantes circundantes. Por lo tanto, el ciclo de los residuos que terminan en este tipo de basurales es el siguiente: primero, la generación de residuos (orgánicos e inorgánicos); segundo, su manipulación y almacenamiento en origen (esta fase es la más desfavorable respecto a la salud pública en los asentamientos informales), y tercero, la recolección, la cual es precaria o nula, dependiendo del sistema de infraestructura vial del sector (D'hers 2013).

En el caso de la ciudad de La Plata, un estudio previo realizado por el grupo de investigación de pertenencia (Discoli et al. 2013) encontró que la gran mayoría de basurales identificados en la región son de bajo impacto. Sin embargo, algunas localizaciones puntuales presentan basurales de alto impacto que, por su extensión (ubicación y superficie que ocupa), volumen (cantidad de basura), composición (orgánica, inorgánica o patológica) y ubicación (lugar

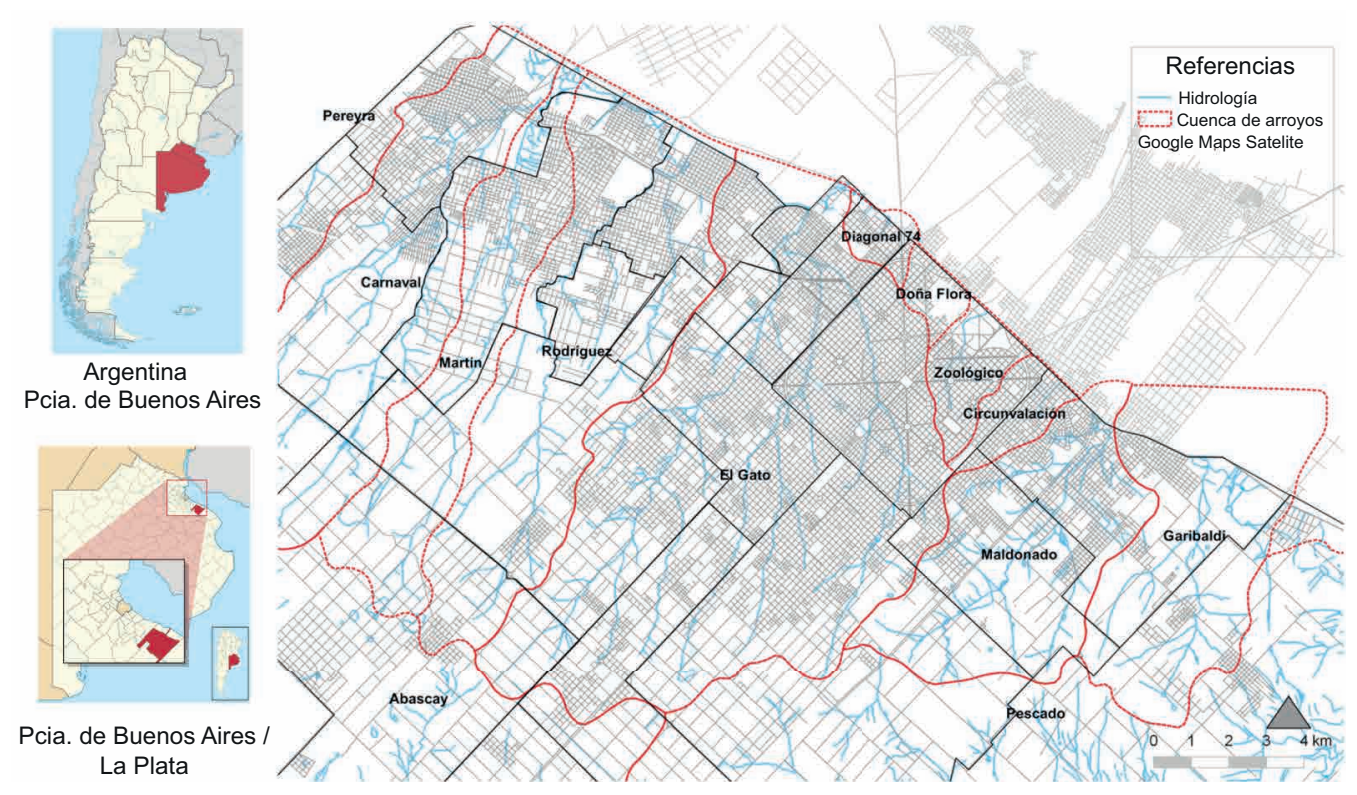

Fig. 1. Ciudad de La Plata: casco urbano y delegaciones municipales. 
donde se encuentra) respecto a ciertos contextos, son calificados de esta manera. ${ }^{6} \mathrm{Si}$ bien se trata de basurales urbanos de baja toxicidad, siempre existe la posibilidad de que se sumen desechos de otro origen (industrial o patológico), ya que no se llevan a cabo acciones concretas de supervisión o erradicación. En estos casos, el factor de permanencia modificaría el grado de inocuidad de los residuos vertidos, es decir, cuanto más permanezcan activos dichos basurales mayor será su grado de impacto. En la figura 2 se muestran los basurales identificados y categorizados en trabajos anteriores.

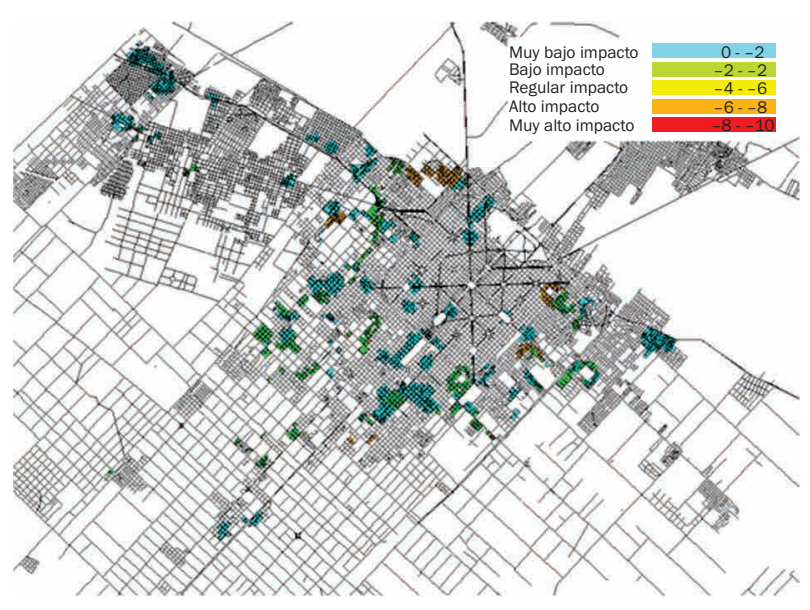

Fig. 2. Clasificación de basurales según estudios previos.

\section{Información de base y su sistematización}

El análisis y recolección de la información utilizada proviene de diversas fuentes. Se ha trabajado sobre datos numéricos, estadísticos, cartográficos y fotográficos provenientes de diferentes organismos. La primera etapa del trabajo se realizó a partir de la superposición de información georreferenciada con un sistema de información geográfica (SIG; software gratuito QGIS v. 2.28) para la obtención de la cartografía temática. Luego, con los resultados obtenidos, se realizó un análisis estadístico sobre la superficie y población afectada. Por último, los casos críticos fueron analizados y desarrollados por medio de un registro fotográfico. A continuación, se detalla el tipo, la fuente, el año y la etapa de trabajo de cada una de las fuentes utilizadas (Cuadro I).

En trabajos anteriores (Esparza 2015) se trabajó con la clasificación de los basurales en función de su extensión y composición. En este trabajo en particular su clasificación se hizo en función de su posible erradicación, es decir, de la posibilidad a ser removidos en el transcurso del tiempo. Por lo tanto, se puede hacer mención de aquellos basurales de alto impacto o "difícil erradicación" y aquellos de "fácil erradicación". En primera instancia, se les identifica y evalúa en sectores con "asentamientos precarios" y aquellos con riesgo hídrico. Se entiende que cualquiera de estas dos situaciones, independientes o superpuestas, genera una mayor afectación y vulnerabilidad

CUADRO I. PROCEDENCIA DE LA INFORMACIÓN UTILIZADA PARA ESTA INVESTIGACIÓN.

\begin{tabular}{llllll}
\hline \multicolumn{1}{l}{ Dato } & Tipo & Fuente & Año & Etapa \\
\hline 1 & Ubicación y extensión de basurales & SHP (QGIS) & IIPAC & 2012 & Etapa 1 \\
2 & Áreas inundables & SHP (QGIS) & CISAUA & 2006 & Etapa 2 \\
3 & Asentamientos, villas, otros & SHP (QGIS) & RPPVAP & 2015 & Etapa 2 \\
4 & Población total, población menor a 14 años, & & & \\
& población mayor a 65 años & SHP (QGIS) & INDEC & 2010 & Etapa 2 \\
5 & Población y áreas afectadas por basurales & Excel & Elaboración propia & 2019 & Etapa 3 \\
6 & Registro fotográfico de basurales & SHP (QGIS), web & Informe sobre basurales & 2018 & Etapa 4 \\
7 & Registro fotográfico de basurales & Fotografías, web & Diario El Día & 2018 & Etapa 4 \\
\hline
\end{tabular}

SHP: shape, QGIS: sistema de información geográfica, IIPAC: Instituto de Investigaciones y Políticas del Ambiente Construido, CISAUA: Centro de Investigaciones en Suelos y Agua de uso agropecuario, RPPVAP: Registro Público Provincial de Villas y Asentamientos Precarios, INDEC: Instituto Nacional de Estadísticas y CensosNota: El Día es el diario de mayor tirada en la ciudad de La Plata (de acceso libre y gratuito).

6 Se considera basurales de alto impacto a aquellos que, teniendo en cuenta elementos inmediatos urbanos permeables a una mayor vulnerabilidad, conviven con funciones tales como escuelas, establecimientos de salud, comederos, espacios públicos, etc., en una misma área afectada. 
en cuanto a la situación de origen. Por otro lado, se calcula la población afectada en relación con rangos etarios, ${ }^{7}$ debido a su capacidad de hacer frente a las inclemencias contaminantes de los mismos.

\section{Tipos de basurales \\ Basurales de difícil erradicación}

El principal conflicto ambiental en la gestión de los residuos urbanos es la creciente acumulación transitoria o crónica de medianos y grandes basurales. La ciudad de La Plata no escapa a esa tendencia general sobre la composición y conformación de los grandes basurales a cielo abierto y/o clandestinos. En la región se observa una serie de basurales denominados "macrobasurales de difícil erradicación", diseminados en importantes montículos dentro del partido, más precisamente por fuera del casco fundacional y bordeando los arroyos de la zona. Existen servicios del municipio que periódicamente los detectan y trasladan hacia el actual relleno sanitario del CEAMSE, pero una vez removidos, esos lugares son nuevamente utilizados como vertederos (Fig. 3).

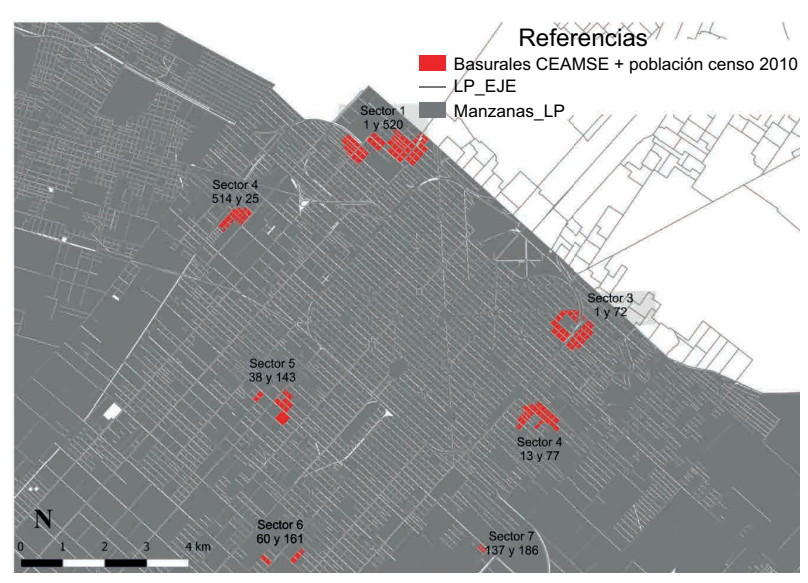

Fig. 3. Ubicación y extensión de los basurales de difícil erradicación.

Este tipo de basurales se considera de volumen variable, pero frecuentes en su proliferación, ya que forman parte del sistema ilegal de vuelco de residuos. Del mismo modo, por su localización y radio de influencia (asociada a su origen y grado de inocuidad: urbano, patológico, peligroso, etc.), estos basurales se consideran críticos respecto a su afectación (Esparza 2016). Su proliferación y constante resurgimiento se debe a la eliminación de residuos húmedos domiciliarios por parte de los habitantes, pero principalmente a los vuelcos clandestinos producidos por vuelcos clandestinos realizados en terrenos privados.. Algunos de éstos, pese a los esfuerzos por erradicarlos, son conocidos por su tamaño y cronicidad, tal es el caso del ubicado en calle 1 y 520, entre las vías del ferrocarril y el Mercado Comunitario (Fig. 3, sector 1).

\section{Basurales de fácil erradicación}

En la Región de La Plata se puede observar una serie de pequeños basurales que pueden denominarse de fácil erradicación debido a que se encuentran principalmente constituidos por pequeños montículos de basura, no perdurables en el tiempo, que se ubican en un punto fijo (esquina) o superficie (manzana o terreno baldío). Este tipo de basurales (principalmente de residuos orgánicos, papeles, botellas y residuos no habituales como ramas y escombros) se consideran de volumen variable, ya que son erradicados esporádicamente. Del mismo modo, por su localización y radio de influencia (asociada a su origen y grado de inocuidad) estos basurales se consideran de poca criticidad respecto a su afectación. Sin embargo, se encuentran distribuidos homogéneamente en todo el territorio, lo que implica un importante porcentaje de población afectada (Fig. 4).

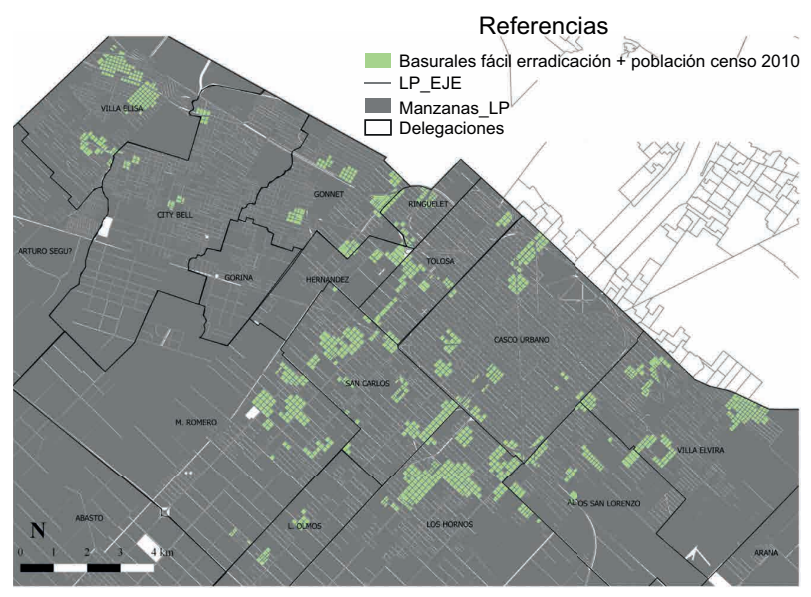

Fig. 4. Ubicación y extensión de los basurales de fácil erradicación (vector fijo).

7 De acuerdo con el último censo nacional se ha caracterizado a la población menor de 14 años y mayor de 65 años por ser altamente vulnerable en relación con su capacidad motora y de acción (INDEC 2010). 


\section{RESULTADOS Y DISCUSIÓN}

Como se mencionó anteriormente, la ciudad de La Plata se encuentra ubicada sobre un territorio cuya concavidad incide sobre la retención o la lenta/muy lenta evacuación del agua proveniente de lluvias o de aguas arriba (Karol y San Juan 2018); por lo tanto, el $45 \%$ de los basurales estudiados se encuentran sobre un entorno de riesgo hídrico.

A partir de estudio previos (Esparza 2015) se conoce que estos sectores se encuentran doblemente vulnerados, no sólo por la existencia del basural en sí mismo, sino por encontrarse en áreas de riesgo hídrico; por lo tanto, se considera de importancia conocer el estado de la población afectada tanto por basurales como por inundaciones, ya que ambos parámetros contribuyen al detrimento de las condiciones de vida y están muy relacionados entre sí en el contexto estudiado. En tal sentido, la situación de riesgo y contaminación se incrementa.

Se consideran como áreas inundables (AI) o con riesgo hídrico (RI) a aquellas propensas a sufrir inundaciones, tanto por causas naturales como antrópicas. Entre las causas naturales se pueden mencionar el exceso de precipitaciones y el aumento en el caudal de ríos y arroyos, así como su desagüe natural. Entre las antrópicas, la deforestación, el asfaltado, la canalización y la ocupación de los cauces. En tal sentido, la acumulación de basura en los cursos de agua retrasa el escurrimiento.

Por otro lado, el grado de impacto y contaminación de los basurales es proporcional a la cantidad y características de la población afectada. Para tal caso, se han estudiado los asentamientos precarios, villas y otros asentamientos (AVO) (RPPVAP 2015), lo cual permite obtener información integrada de origen social y catastral de la población de bajos recursos ubicada en asentamientos informales.

En la figura 5 se observa la superficie afectada por basurales que se encuentran en RI. El sector de mayor afectación es el ubicado en la delegación de San Carlos con una extensión de 66 ha; le siguen en orden descendente los sectores José Hernández, Gonnet y Villa Elisa (rango 40-53 ha). Esta situación, permite determinar cuáles son las áreas que se encuentran no solamente contaminadas por basurales, sino en riesgo de inundaciones, incrementando la inhabitabilidad de los sectores afectados por ambos criterios.

Por otro lado, en la figura 6 se observan aquellos asentamientos informales ubicados en torno a basurales. Tanto en las referencias como en la imagen, se puede observar la cantidad de familias afectadas. En primer lugar, se encuentra los asentamientos

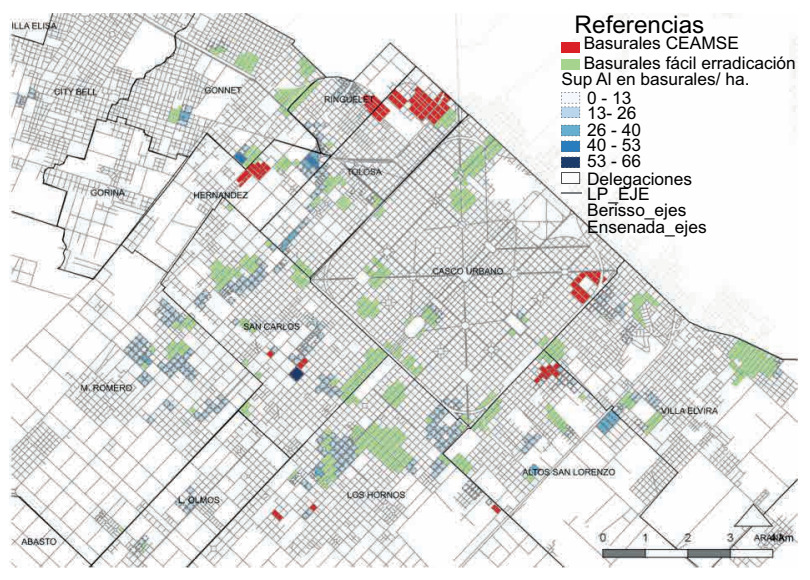

Fig. 5. Superficie en hectáreas de áreas inundables sobre basurales.

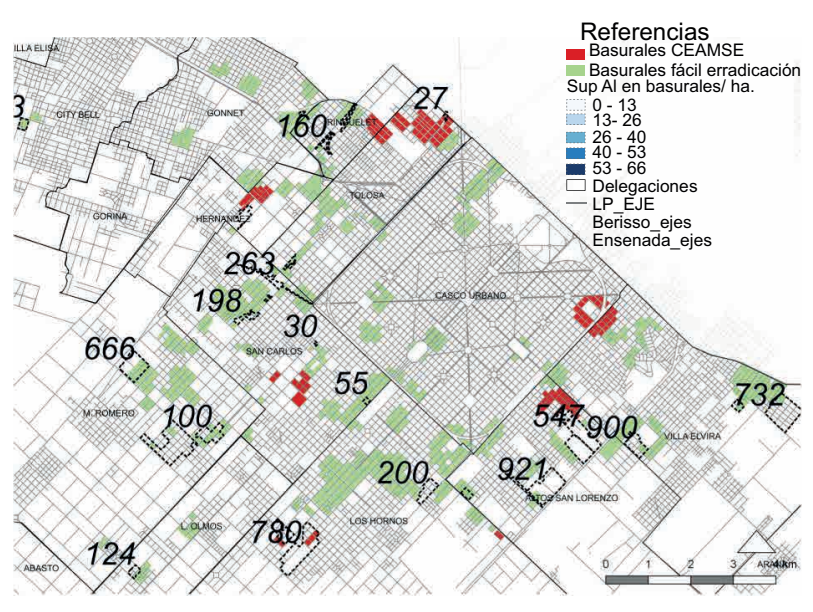

Fig. 6. Cantidad de familias que habitan en asentamientos precarios ubicados en torno a basurales.

ubicado en la delegación de Altos de San Lorenzo con 921 familias afectadas. Luego, continúan el de Villa Elvira, con 900 familias afectadas, y el de Los Hornos, con 780 familias.

En el Cuadro II se presenta de manera sintética la superficie (en hectáreas) de áreas inundables y la cantidad de familias en asentamientos informales afectadas por basurales. De forma paralela se analiza la población afectada por basurales según los rangos etarios y su cercanía o no a las áreas con riesgo hídrico y de asentamientos informales. Cabe aclarar que el código de ordenamiento urbano vigente no contempla un modelo de ocupación del suelo explícito. En este contexto, la población ha quedado desprotegida respecto a la implicancia ambiental de los basurales y las inundaciones; por el contrario, a pesar de que se conoce la situación geomorfológica 
e hidráulica de la región, se permite la ocupación de estas áreas. Simplemente se localizan las actividades industriales, urbanas, agrícolas, etc., sin previsión de sus consecuencias (UNLP 2014-2016).

CUADRO II. TABLA DE VALORES.

\begin{tabular}{lrc}
\hline & $\begin{array}{c}\text { AI } \\
\text { (ha) }\end{array}$ & $\begin{array}{c}\text { AVO } \\
\text { Cantidad de } \\
\text { familias/ha }\end{array}$ \\
\hline Basurales de difícil erradicación & 52.36 & $101 / 2146$ \\
Basurales de fácil erradicación & 518.73 & $305 / 6783$ \\
\hline
\end{tabular}

AI: áreas inundables, AVO: asentamientos, villas y otros.

\section{Basurales de difícil erradicación}

Los basurales de difícil erradicación o grandes basurales están compuestos principalmente por material orgánico como restos de comida, papel y cartón; luego, los inorgánicos como metales, vidrio y plástico. ${ }^{8}$ En la figura 7 se observa la ubicación de los basurales de difícil erradicación en torno a sectores con RI y AVO. En el cuadro III se presenta una serie de atributos de los basurales identificados, entre los cuales se pueden mencionar: a) extensión (superficie en metros cuadrados), b) proximidad a áreas con RI y AVO (distancia en metros) y c) cantidad de población afectada según rangos etarios. De esta manera, a modo comparativo, se ha podido definir cuáles son los basurales de mayor afectación.

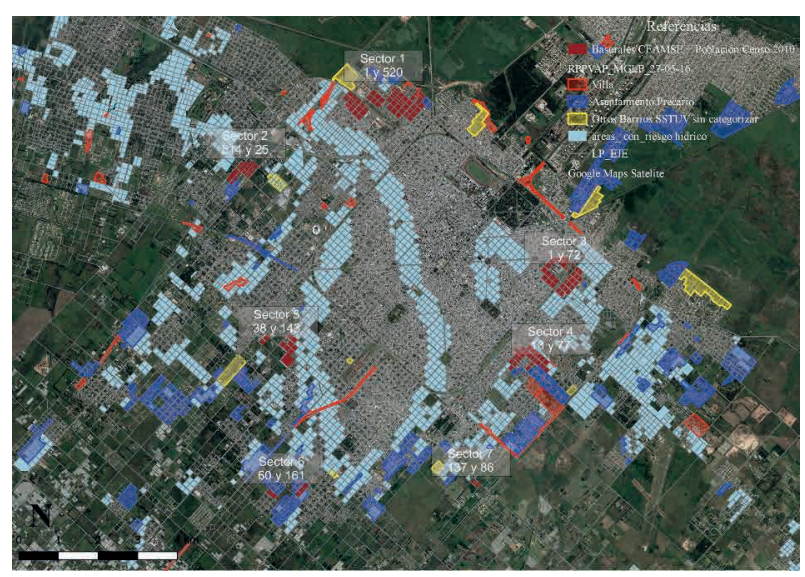

Fig. 7. Basurales de la Coordinación Ecológica Área Metropolitana Sociedad del Estado (CEAMSE) y su ubicación respecto a zonas con riesgo hídrico y asentamientos precarios.

En consecuencia, se observa que los sectores más críticos, por su extensión y ubicación, son el 1 (Ringuelet), el 3 (Villa Elvira) y el 4 (Altos de San Lorenzo). En una situación intermedia, encontramos a los sectores 2 y 5 , y, por último, a los sectores 6 y 7 (Fig. 7). A continuación se detalla cada uno de los basurales de mayor afectación.

\section{Sector 1}

En relación con su extensión espacial y cantidad de habitantes afectados, el basural ubicado en el Sector 1 (de Avenida 122 a Calle 4 y de Calle 517 a

CUADRO III. SUPERFICIE Y CANTIDAD DE HABITANTES AFECTADOS POR BASURALES DE DIFÍCIL ERRADICACIÓN

\begin{tabular}{|c|c|c|c|c|c|c|c|}
\hline & & $\begin{array}{c}\text { Superficie } \\
\text { Afectada } \\
\left(\mathrm{m}^{2}\right)\end{array}$ & $\begin{array}{c}\text { Distancia a } \\
\text { RI/AVO } \\
\text { (m) }\end{array}$ & $\begin{array}{c}\text { Habitantes } \\
\text { afectados } \\
\text { (0 a } 14 \text { años })\end{array}$ & $\begin{array}{c}\text { Habitantes } \\
\text { afectados } \\
\text { (15 a } 65 \text { años) }\end{array}$ & $\begin{array}{c}\text { Habitantes } \\
\text { afectados } \\
\text { (+ de } 65 \text { años })\end{array}$ & $\begin{array}{l}\text { Habitantes } \\
\text { afectados } \\
\text { (total) }\end{array}$ \\
\hline \multirow{7}{*}{ 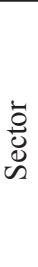 } & 1 & 698976 & 100 & 1063 & 3059 & 662 & 4784 \\
\hline & 2 & 158846 & 100 & 70 & 194 & 29 & 293 \\
\hline & 3 & 361729 & 500 & 429 & 1642 & 357 & 2428 \\
\hline & 4 & 262275 & 0 & 429 & 1173 & 212 & 1723 \\
\hline & 5 & 194686 & 700 & 171 & 443 & 50 & 664 \\
\hline & 6 & 68939 & 0 & 50 & 86 & 5 & 141 \\
\hline & 7 & 19029 & 500 & 3 & 4 & 0 & 7 \\
\hline
\end{tabular}

RI: riesgo hídrico; AVO: asentamientos, villas y otros

8 En este trabajo se estima que la conformación de los basurales de difícil erradicación es la siguiente: papel y cartón, $14.58 \%$; textiles, $4.10 \%$; plásticos, $22.78 \%$; vidrio, $3.80 \%$; metales, $2.03 \%$; otros materiales, $19.59 \%$, y material orgánico, $33.12 \%$ (alimenticios, $30.14 \%$, y residuos de poda o ramas, $2.98 \%$ ). Su volumen se define como variable, ya que si bien son erradicados, por medio de vuelcos clandestinos su volumen aumenta nuevamente (SGAyDS 2018). 


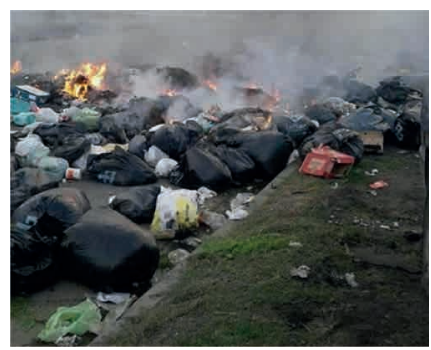

Calles 520 y119

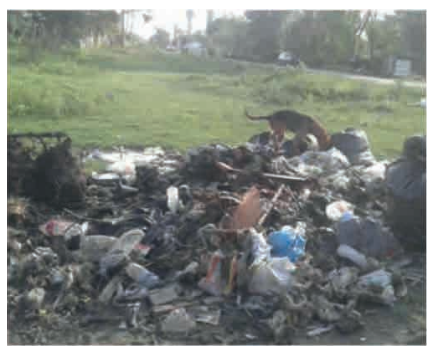

Calles 520 y 122

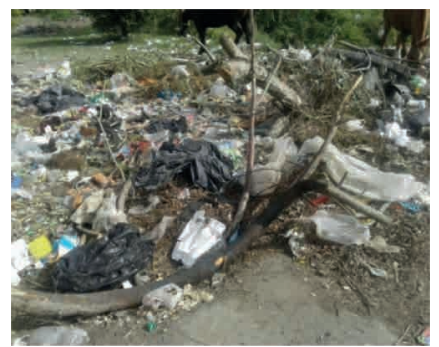

Calle 122 entre 527 y 529

Fig. 8. Registro fotográfico de los basurales a cielo abierto del Sector 1.

Calle 529) es el que genera mayor afectación. Con un total de 4784 habitantes afectados e incluso con una fuerte presencia de asentamientos informales en el área, se le considera el basural a cielo abierto que genera mayor vulnerabilidad en la región. En este sentido, hay 211 familias en villas, 387 familias en asentamiento precarios y 48 familias en otro tipo de asentamiento (RPPVA 2015). Paralelamente, cabe destacar que el sector se ubica en un interfluvio principal, con cotas de inundación que varían de 20 a $30 \mathrm{msnm}$ - las cotas más altas del Partido (UNLP 2014-2016) - , lo cual indica que es una zona poco inundable. El basural de este sector se encuentra a 500 $\mathrm{m}$ del sector inundable (Cuadro III). En la Figura 8 se observa un registro fotográfico del basural del Sector 1.

\section{Sector 3}

Otros sectores fuertemente afectados por basurales son los 3 y 4 , con 361729 y $262275 \mathrm{~m}^{2}$ de extensión, respectivamente. El basural del Sector 3, ubicado en inmediaciones del Hospital Interzonal de Agudos General San Martin afecta aproximadamente a 2428 habitantes. Está ubicado en las inmediaciones de la planicie de inundación del Arroyo Maldonado, lo que genera una situación de vulnerabilidad social y ambiental respecto a su ubicación en áreas con riesgo hídrico (Cuadro III). Este sector de la ciudad se caracteriza por su precario estado respecto a los sistemas básicos de infraestructura. No sólo hay deficiencias en la recolección de residuos, sino también en obras de desagüe y pavimentación, lo cual genera anegamientos y, principalmente, áreas no aptas para el asentamiento humano.

La característica principal de los basurales de esta zona es que se encuentran ubicados en esquinas o espacios verdes (como ramblas o terrenos baldíos) y presentados en montículos medianos y grandes, principalmente debido a la disposición vecinal por la falla en el sistema de recolección. Si se tiene en cuenta la cantidad de población afectada, su ubicación en sectores de inundación y su ubicación con el hospital mencionado, su grado de afectación aumenta considerablemente, identificándolo como uno de los sectores de mayor afectación en la región. La Figura 9 presenta una serie de imágenes de los basurales de este sector.

\section{Sector 4}

La situación del sector 4 es compleja debido a la cercanía del basural a áreas con RI y AVO. En tal sentido, las características del terreno de dicho
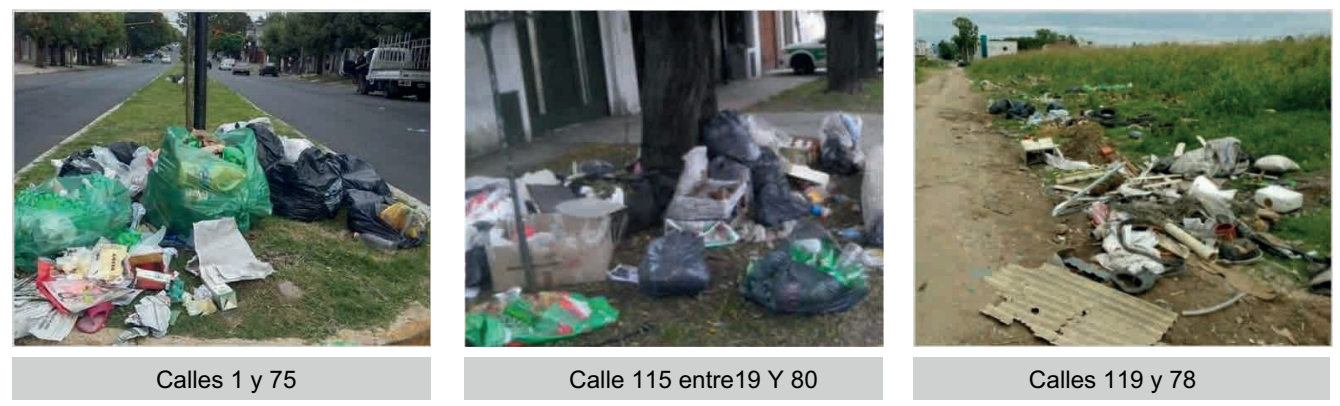

Fig. 9. Registro fotográfico de los basurales a cielo abierto del Sector 3. 
sector - profundamente alterado por la actividad del hombre- derivan en permanentes conflictos hidráulicos, los cuales se agravan por la baja permeabilidad del suelo del sector. El escurrimiento natural de la zona, que se produce a través de los arroyos El Pescado y Maldonado, provoca desbordes ocasionales e inconvenientes para los habitantes asentados en sus inmediaciones. Como se puede observar en la figura 7, este sector (en color celeste) se encuentra fuertemente afectado por inundaciones.

Por otro lado, según el Registro Público Provincial de Villas y Asentamientos Precarios (RPPVAP 2015), en el Partido de La Plata hay un total de 88 asentamientos informales localizados tendencialmente hacia el eje sudeste y sudoeste (sectores 3 y 4), caracterizados por tener problemáticas ambientales y económicas, así como de crecimiento urbano, accesibilidad y abastecimiento de servicios básicos de infraestructura (Rodríguez-Tarducci 2018). Este sector se destaca por la falla en la recolección de residuos, lo cual genera un área importante de acumulación de basurales, cuya composición es principalmente de tipo mixta (basura orgánica, inorgánica, escombros y ramas, entre otros) sin separación ni clasificación de residuos.

En tal sentido, el Sector 4, con un total de 1724 habitantes asentados en los márgenes del basural, es uno de los más críticos. Asimismo, en el área se encuentran ubicadas 958 familias en villas, 1981 familias en asentamiento precarios y 60 familias en otros tipos de asentamientos, lo que indica una situación de vulnerabilidad urbana, territorial y ambiental compleja con relación al resto de los sectores identificados.

Por otro lado, en otro sector circundante del área (inmediaciones de la Calle 90 y la Avenida 137) se ubica un basural de grandes dimensiones. Aquí, la falta de regulación respecto a la eliminación de desechos ha generado una constante situación de vulnerabilidad en la zona provocando un importante basural a cielo abierto (Fig. 10).

\section{Basurales de fácil erradicación}

La proliferación de pequeños montículos de residuos en toda el área estudiada se debe principalmente a la falla o falta de recolección, barrido y limpieza de la región. Si bien la municipalidad cuenta con un sistema específico de recolección de días y horarios establecidos para cada área de la ciudad, éste se considera ineficiente en su actividad. Este tipo de basurales se puede detectar con facilidad al transitar cotidianamente por la ciudad, lo que deja en evidencia la inacción municipal antes mencionada. Como consecuencia, barrios enteros padecen una recolección deficiente, exponiendo a los vecinos a un gran riesgo ambiental que afecta su salud y su seguridad.

Estos montículos o pequeños basurales esparcidos en toda la región son el principal resultado del incremento de los malos hábitos de la población, que se expresa en acciones generalizadas como sacar la basura fuera de horarios establecidos, arrojar residuos en la vía pública y/o depositar los mismos en sectores alejados al domicilio, como esquinas, terrenos baldíos y arroyos, entre otros. Esta situación, producto de campañas insuficientes para la concientización del problema de la basura, se encuentra complementada con la falla en la recolección de éstos.

El crecimiento y concentración de la población en áreas suburbanas, la escasa e ineficiente infraestructura de calles (pavimento, zanjeo, desagües pluviales, etc.) ha incrementado esta problemática. Asimismo, el aumento en la cantidad de basura generada por persona, comercio o industria, y los cambios en hábitos de consumo, han acrecentado la generación de residuos de envases y embalaje, y generado problemáticas en su posterior disposición. En el casco urbano este servicio queda cubierto en parte por el cirujeo, pero en la periferia su acumulación genera

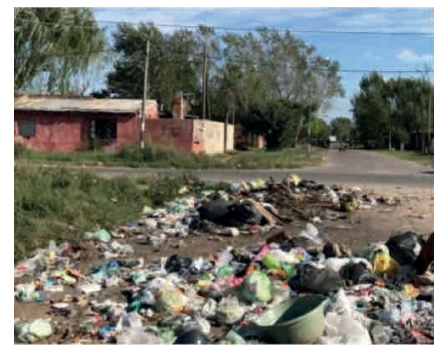

Calles 16 y 81

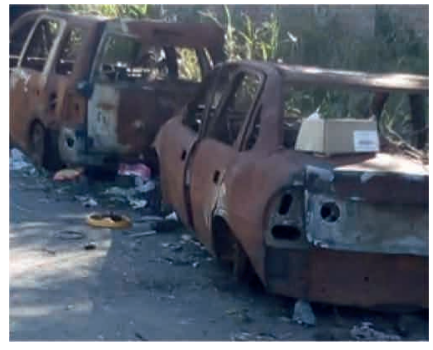

Calle 23bis entre79 y 80

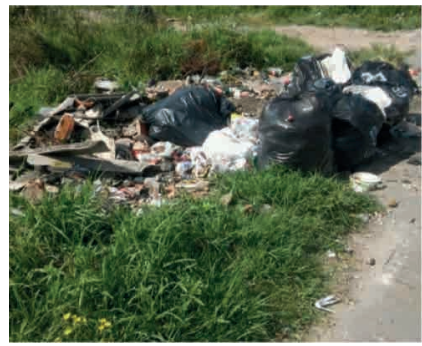

Calles 25 y 83

Fig. 10. Registro fotográfico de los basurales a cielo abierto del Sector 4. 
focos de desarrollo de basurales y degradación de los arroyos.

Por otro lado, como en el caso de los basurales de difícil erradicación, su ubicación y extensión se ha analizado junto con información relacionada a áreas de RI y con aquella que describe la ubicación y situación de AVO en la región. El cruce de esta información permite determinar cuáles son las áreas de mayor afectación y, en consecuencia, determinar su posterior erradicación en el corto, mediano o largo plazo. Como se dijo anteriormente, la basura que se encuentra en áreas críticas inundables o en torno a población de bajos recursos, genera una situación de mayor vulnerabilidad para la vida humana (Fig. 11).

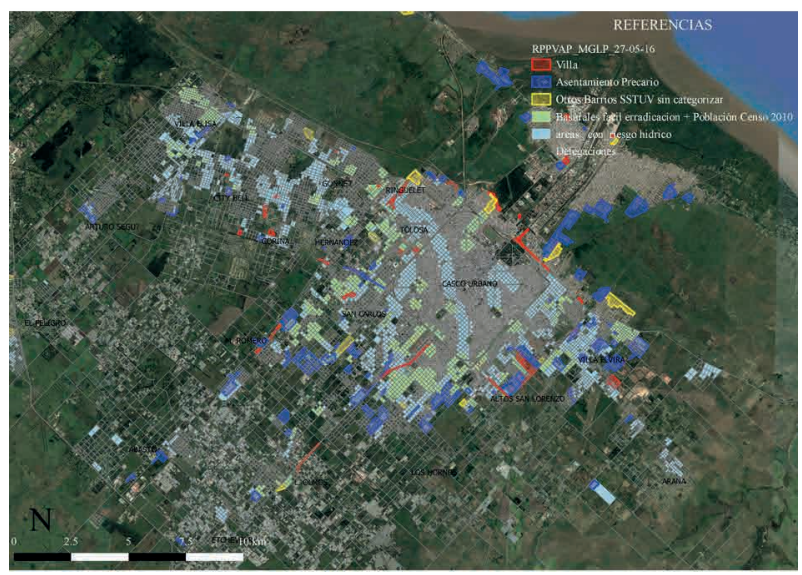

Fig. 11. Basurales de fácil erradicación y su ubicación respecto a zonas con riesgo hídrico y asentamientos precarios, villas y otros
A diferencia del análisis sobre los basurales de difícil erradicación, estudiados por sectores o áreas de afectación, en este caso el análisis se ha realizado en toda la región, identificando la situación particular de cada una de las delegaciones de la ciudad. Del mismo modo, se han identificado y caracterizado aquellas afectadas también por inundaciones (se presentan en color celeste) y ubicadas en torno a asentamientos precarios (se presenta en colores rojo, azul y amarillo) (Fig. 11).

En el siguiente cuadro se observa la superficie afectada por basurales en las delegaciones de la ciudad de La Plata, así como su cercanía con áreas de RI y AVO. La cantidad de población afectada se distribuye según grupos etarios, ya que los menores de 14 y mayores de 65 años se consideran de mayor vulnerabilidad (INDEC 2010), lo que permite identificar cuáles son los sectores o delegaciones más vulnerables y, en consecuencia, determinar medidas de mejoramiento o mitigación (Cuadro IV).

Asimismo, se destaca que en función de las características estudiadas, las delegaciones se encuentran en situaciones muy dispares entre ellas, no sólo por la extensión de los basurales y su ubicación sino por la cantidad de habitantes afectados. Por ello, el cuadro IV, además exponer la situación, permite comparar y medir los resultados en cada una de ellas. En primer lugar, es evidente que el basural de mayor extensión que afecta a un mayor número de habitantes se encuentra en la delegación de Los Hornos. Por otro lado, son notables los rangos de población total afectada. Se observan tres rangos definidos con diferencias importantes de cantidad en cada uno de ellos: 1) Los

CUADRO IV. SITUACIÓN DE LOS BASURALES DE FÁCIL ERRADICACIÓN (VECTOR FIJO).

\begin{tabular}{|c|c|c|c|c|c|c|c|}
\hline Delegación & $\begin{array}{l}\text { Superficie } \\
\text { afectada } \\
\text { (ha) }\end{array}$ & $\begin{array}{c}\text { RI } \\
\text { (a } 0 \mathrm{~m})\end{array}$ & $\begin{array}{c}\text { AVO } \\
(\mathrm{a} 0 \mathrm{~m})\end{array}$ & $\begin{array}{l}\text { Habitantes } \\
\text { afectados } \\
\text { (0 a } 14 \text { años })\end{array}$ & $\begin{array}{l}\text { Habitantes afec- } \\
\text { tados } \\
\text { (15 a } 65 \text { años) }\end{array}$ & $\begin{array}{c}\text { Habitantes } \\
\text { afectados } \\
\text { (+ de } 65 \text { años })\end{array}$ & $\begin{array}{l}\text { Habitantes } \\
\text { afectados } \\
\text { (total) }\end{array}$ \\
\hline Los Hornos & 223.70 & Sí & Sí & 2833 & 8245 & 1417 & 12495 \\
\hline San Carlos & 163.68 & Sí & Sí & 1948 & 4919 & 719 & 7586 \\
\hline Villa Elvira & 140.43 & Sí & Sí & 1871 & 4763 & 635 & 7269 \\
\hline Tolosa & 66.72 & Sí & Sí & 870 & 2442 & 379 & 3691 \\
\hline A. de San Lorenzo & 71.93 & Sí & Sí & 910 & 2305 & 299 & 3514 \\
\hline Villa Elisa & 122.20 & Sí & Sí & 744 & 2286 & 406 & 3436 \\
\hline Melchor Romero & 111.47 & Sí & Sí & 1069 & 2008 & 122 & 3227 \\
\hline Gonnet & 85.82 & Sí & Sí & 548 & 1580 & 239 & 2367 \\
\hline Ringuelet & 59.71 & Sí & Sí & 505 & 1304 & 220 & 2029 \\
\hline Lisandro Olmos & 47.10 & Sí & Sí & 159 & 397 & 46 & 602 \\
\hline City Bell & 25.98 & Sí & Sí & 141 & 359 & 54 & 554 \\
\hline Hernández & 26.95 & Sí & Sí & 82 & 225 & 30 & 337 \\
\hline
\end{tabular}

Fuente: elaboración propia.

RI: riesgo hídrico; AVO: asentamientos, villas y otros. 


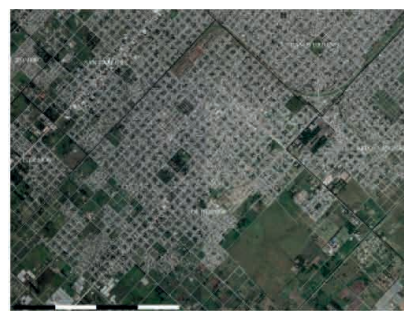

Imagen satelital del sector (Google 2018)

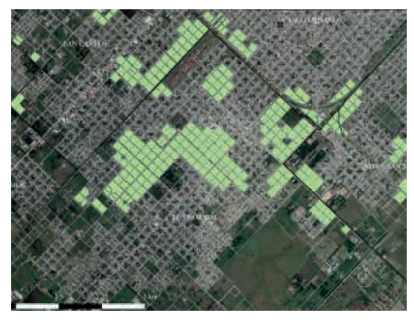

Basurales de fácil erradicación proyectados sobre imagen satelita (Google 2018)

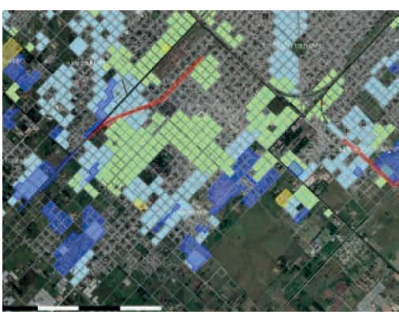

Basurales de fácil erradicación, áreas con riesgo hídrico, y asentamientos, villas y otro tipo de asentamientos proyectados sobre imagen satelital (Google 2018)

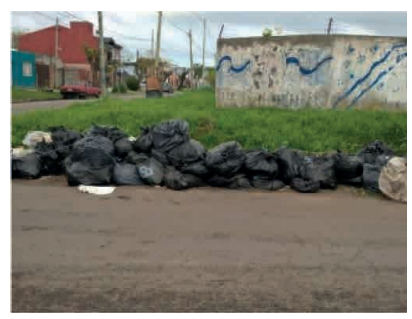

Registro fotográfico de basurales en la zona, calles 64 y 151 (Diario El Día 28/09/18)

Fig. 12. Basurales de fácil erradicación en la Delegación de Los Hornos.

Hornos, San Carlos y Villa Elvira; 2) Tolosa, Altos de San Lorenzo, Villa Elisa, Melchor Romero, Gonnet y Ringuelet, y 3) Lisandro Olmos, City Bell y Hernández. A continuación se hace una breve descripción de las delegaciones con mayor afectación de acuerdo con el número de población total afectada.

En la delegación de Los Hornos (Fig. 12) existe una superficie afectada por basurales de 223.70 ha y una población total afectada de 12495 habitantes. En este sector, en torno a los basurales identificados, se puede encontrar una serie de asentamientos precarios que albergan a 2503 familias en una superficie total de 136.3 ha. En sectores de villas miseria se ubican 228 familias y en otro tipo de asentamientos informales 110 familias (RPPVAP 2015). Los sectores de basurales que se encuentran en contacto con este tipo de asentamientos se encuentran en las inmediaciones de las siguientes intersecciones: a) calles 58 y 155, b) calles 70 y 141, y c) calles 81 y 139 (Los Hornos).

Por otro lado, en cuanto a la ubicación de los basurales en áreas con riesgo hídrico, cabe mencionar que se encuentran en los cauces de los Arroyos Pérez y Regimiento, generando una situación poco favorable para los asentamientos humanos. Según el mapa, los sectores de mayor criticidad son aquellos comprendidos entre: a) las calles 146 a 157 y 54 a 62 (sector de mayor extensión, de 42.64 ha), b) las calles 31 a 137 y 66 a 72 (con una extensión de 36.09 ha).

En la delegación de San Carlos se localiza una superficie afectada por basurales de 163.68 ha y una población total afectada de 7586 habitantes. En tal sentido, se pueden observar en el mapa resultante sectores de afectación por la superposición de basurales, asentamientos informales y áreas de riesgo hídrico (Fig. 13). Por ejemplo, la zona comprendida entre las calles 134 a 143 y 522 a 546, atravesada por el Arroyo del Gato, se encuentra afectada fuertemente por inundaciones. Del mismo modo, en ese sector se encuentra la villa denominada Toba o Barrio Toba, donde conviven alrededor de 198 familias carentes de servicios básicos de infraestructura, como el sistema de pavimentación que impide el ingreso del camión recolector de residuos. En el sector también se ubica un asentamiento precario de menor extensión, el cual alberga a 40 familias y cuenta con una cobertura parcial de servicios básicos (RPPVAP 2015).

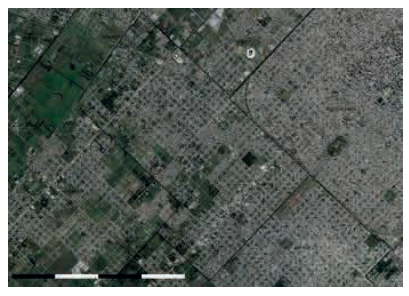

Imagen satelital del sector (Google 2018)

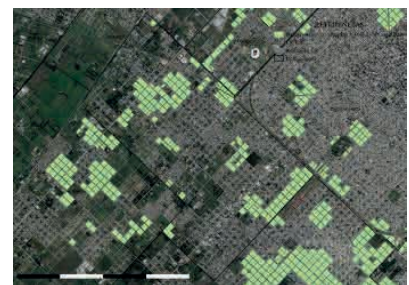

Basurales de fácil erradicación proyectados sobre imagen satelital (Google 2018)

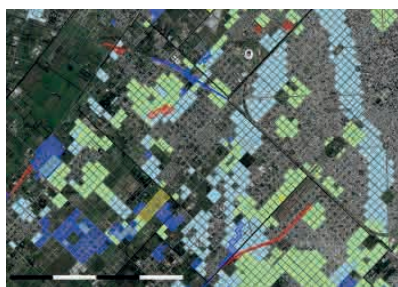

Basurales de fácil erradicación, áreas con riesgo hídrico, $y$ asentamientos, villas y otro tipo de asentamientos proyectados sobre imagen satelital (Google 2018)

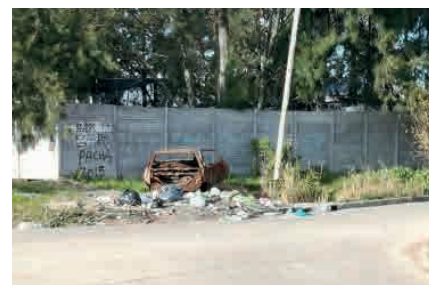

Registro fotográfico de basurales en la zona, calles 43 y 141 (Diario El Día 23/06/18)

Fig. 13. Basurales de fácil erradicación en la Delegación de San Carlos. 


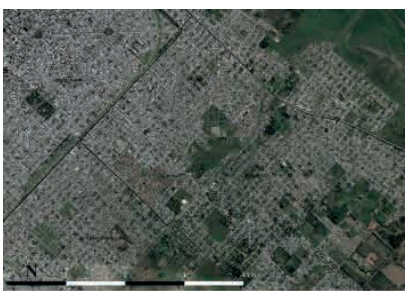

Imagen satelital del sector (Google 2018)

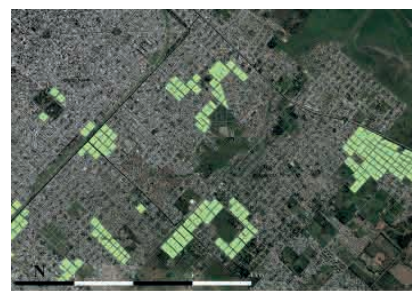

Basurales de fácil erradicación proyectados sobre imagen satelital (Google 2018)

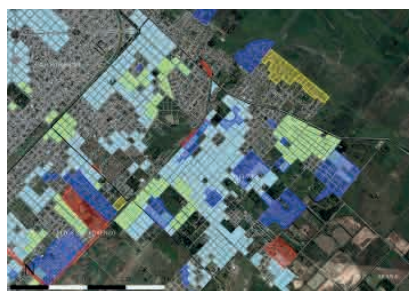

Basurales de fácil erradicación, áreas con riesgo hídrico, y asentamientos, villas y otro tipo de asentamientos proyectados sobre imagen satelital (Google 2018)

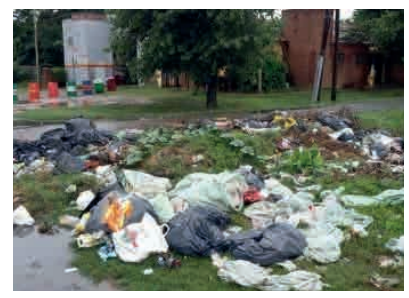

Registro fotográfico de basurales en la zona, Ruta 11 y Calle 601 (Diario El Día 13/02/17)

Fig. 14. Basurales de fácil erradicación en la Delegación de Villa Elvira.

Otra de las grandes áreas afectadas por basurales y atravesada por áreas con riesgo hídrico es la comprendida de las calles 140 a la 143 y de la 44 a la 50. En la cercanía del sector se encuentran ubicadas 203 familias en un asentamiento precario, lo que genera un área con una importante cantidad de personas afectadas.

En la Delegación de Villa Elvira hay una extensión aproximada de 140.43 ha de basurales de fácil erradicación esparcidos en cuatro sectores: 1) calles 121 a 2 y 75 a $82 ; 2$ ) calles 10 a 13 y 72 a 76 ; 3) calles 90 a 600 y 6 a 13, y 4) Ruta Provincial 11 a Calle 119 y calles 601 a 611 . Asimismo, esta superficie suma un total de población afectada de 7269 habitantes (Fig. 14).

De los cuatro sectores, tres se encuentran afectados por inundaciones, principalmente el ubicado en torno a las calles 121 y 75 . En este caso, el sector se encuentra en el cauce del Arroyo Maldonado, el cual afecta notablemente a la población asentada en la zona. En tal sentido, en el área se ubican 1020 familias en asentamientos precarios, siendo el mayor de ellos el ubicado en torno a las calles 11 y 93 , con cobertura parcial a inexistente de los servicios básicos de infraestructura. Cabe mencionar que este asentamiento corresponde a uno de los grupos poblacionales de mayor vulnerabilidad en la región, no sólo por encontrarse cerca de los basurales mencionados, sino por ubicarse en un sector territorial desprovisto de todo tipo de regulación por parte del Estado en cuanto a gestión e infraestructura.

\section{CONCLUSIONES}

La gestión de RSU se ha transformado en un problema ambiental prioritario. Entre los factores que contribuyen a su proliferación y crecimiento se pueden mencionar los siguientes: el modo de producción de la basura; el modo actual de consumo, que responde al paradigma lineal de usar y desechar; la concentración de la población en centros urbanos; la utilización de bienes materiales de rápido deterioro, y el uso cada vez más generalizado de envases no degradables, entre otros. Esta situación, junto con el desarrollo territorial y demográfico de las ciudades actuales, ha propiciado el origen de sectores de acumulación de residuos, fundamentalmente en tierras vacantes, cavas o asentamientos informales y precarios. Esta conjunción, traducida en basurales de grande y mediana escala, ha incrementado notoriamente la contaminación urbana y ambiental del territorio.

La ciudad de La Plata no escapa a esta realidad. Este trabajo ha permitido identificar, entre otros, tres grandes basurales de difícil erradicación y tres grandes áreas de afectación por basurales de fácil erradicación. La población afectada es de 10040 habitantes respecto a los basurales de difícil erradicación (distribuidos en 106 ha) y de 35907 habitantes con relación a los de fácil erradicación (distribuidos en 1145 ha). Respecto a los basurales en áreas inundables, la superficie asciende a 571 ha, en tanto que los ubicados en los márgenes de asentamientos informales abarcan 406 ha, afectando a 8919 familias. Este grupo poblacional constituye el de mayor riesgo en cuanto a contaminación por basurales, ya que pertenece a un sector de escasos o nulos recursos y necesidades básicas insatisfechas, como acceso a salud y educación.

Por otro lado, el estudio de casos de contaminación grave ha permitido observar los parámetros que influyen desde los basurales: contaminación, segregación, insalubridad, etc., y los que lo hacen hacia los basurales: vuelcos en clandestinidad, toma 
de terrenos baldíos de carácter privado, etc. (D'hers 2013). En primer término, la proliferación de focos y vectores infecciosos, la contaminación aérea en caso de quema, la generación de enfermedades cutáneas y respiratorias en niños y adultos mayores, y la contaminación del agua para consumo y la tierra, donde eventualmente se cultivan alimentos para consumo. En segundo termino, el origen de dichos focos como producto de la falta y falla en la recolección de residuos por parte del Municipio y, además, como vertederos clandestinos de los vecinos circundantes. En este sentido, vale destacar el caso de los basurales que surgen por la conformación de asentamientos informales, desprovistos de infraestructura básica, como pavimentación, zanjeo y recolección de residuos. Aquí, como consecuencia del trabajo realizado por sus habitantes (cartoneo y cirujeo) proliferan grandes y medianos montículos de basura de alto valor contaminante.

Los casos estudiados constituyen una clara muestra de la situación actual de los basurales en la ciudad de La Plata. Sin lineamientos claros y efectivos sobre la gestión integral de los RSU, el territorio platense se encuentra actualmente ante un panorama poco favorable en cuanto a la erradicación o mitigación de éstos, principalmente aquellos de gran envergadura.

En consecuencia, se plantea la necesidad de crear planes integrales de urbanización, con esquemas de infraestructura coherentes con el crecimiento y desarrollo poblacional para evitar la proliferación de nuevos vectores contaminantes. Luego, como base del concepto que plantea la economía circular, ${ }^{9}$ se sugiere reglamentar la reutilización de residuos y el reciclaje a partir de su separación y evaluación, como parte de la actividad cotidiana de la población.

\section{AGRADECIMIENTOS}

Esta investigación fue realizada gracias al financiamiento de Consejo Nacional de Investigaciones Científicas y Técnicas (CONICET) en el marco de un proyecto de investigación personal. Del mismo modo, se agradece la financiación al Instituto de Investigación de Pertenencia por parte de Centro Científico Tecnológico del CONICET como sede doble dependencia. Por último, se agradece a los evaluadores y editores por su tiempo, dedicación y sugerencias.

\section{REFERENCIAS}

Crespo G. (2017). Mapa de reclamos [en línea]. https://www.crespogaston.com.ar/mapa-de-reclamos/ $10 / 09 / 2019$.

D'hers V. (2013). Asentamientos sobre basurales a cielo abierto: explotación, segregación y expulsión en el manejo de los residuos. Delos 6 (16), 1-29.

Discoli C., San Juan G., Martini I., Barbero D., Dicroce L., Ferreiro C., Viegas G. y Esparza J. (2013). Calidad de vida en el sistema urbano: una aproximación teórica y metodológica. EDULP, La Plata, Buenos Aires, Argentina, 196 pp.

Esparza J. (2015). Diagnóstico urbano ambiental en las distintas escalas espaciales de la ciudad. Su aplicación en la ciudad de La Plata. Tesis de Doctorado, Doctorado en Arquitectura y Urbanismo, Facultad de Arquitectura y Urbanismo, Universidad Nacional de La Plata, La Plata, Buenos Aires, Argentina, 298 pp.

Esparza J. (2016). Diagnóstico urbano ambiental en las distintas escalas espaciales de la ciudad. Su aplicación en la ciudad de La Plata. Memorias del $7^{\circ}$ Congreso Luso-Brasilero para el Planeamiento Urbano, Regional, Integrado y Sustentable, Brasilia, Brasil, 5 al 7 de octubre.

Hurtado M., Giménez J., Cabral M., da Silva M., Martinez O., Camilión M., Sanchez C., Muntz D., Gebhard J., Forte L., Boff L., Crincoli A. y Lucesoli H. (2006). Análisis ambiental del Partido de La Plata. Aportes al ordenamiento territorial. Consejo Federal de Inversiones, La Plata, Buenos Aires, Argentina, 141 pp.

INDEC (2010). Censo Nacional de Población, Hogares y Viviendas. Aspectos operativos y metodológicos. Informe. Instituto Nacional de Estadísticas y Censos, Ciudad de Buenos Aires, Argentina, 7 pp.

ISWA (2015). El caso trágico de los basurales. Una amenaza para la salud. Informe del Grupo del Comité Técnico Científico. Informe. International Solid Waste Association, Rotterdam, Países Bajos, 49 pp.

Karol J. y San Juan G. (2018). Saber qué hacer. Construcción de un sistema para la gestión integrada del riesgo hídrico en la región del Gran La Plata. CONICET-

9 'Economía circular' es un concepto económico que se interrelaciona con la sostenibilidad. Su objetivo es que el valor de los productos, los materiales y los recursos (agua, energía) se mantengan en la economía durante el mayor tiempo posible y que se reduzca al mínimo la generación de residuos. 
Universidad Nacional de La Plata, La Plata, Buenos Aires, Argentina, 199 pp.

OECD (2004). Addressing the economics of waste. Organization for Economic Co-operation and Development. París, Francia, 203 pp.

ONU (2019). Programa para el Medio Ambiente. Reunión constitutiva de la coalición voluntaria de gobiernos y organismos pertinentes para el cierre progresivo de los basurales en América Latina y el Caribe. Documento. Organización de las Naciones Unidas, Buenos Aires, Argentina, $23 \mathrm{pp}$.

Rodríguez-Tarducci R. (2018). Asentamientos informales en el Partido de La Plata. Una aproximación a las modalidades de ocupación del territorio. Estudios Socio-Territoriales 1 (23), 119-136.
RPPVAP (2015). Villas y asentamientos precarios. Mapas. Registro Público Provincial de Villas y Asentamientos Precarios, Ministerio de Infraestructura y Servicios Públicos, Buenos Aires, Argentina.

SGAyDS (2018). Informe del estado del Ambiente 2018. Informe. Secretaría de Gobierno de Ambiente y Desarrollo Sustentable, Ciudad de Buenos Aires, Argentina, 190 pp.

UNLP (2014-2016). Las inundaciones en La Plata, Berisso y Ensenada: análisis de riesgo y estrategias de intervención. Capítulo 1: Las inundaciones en la Región Capital. Cartografía temática para el planeamiento. Proyectos de Investigación Orientados. Informe. Universidad Nacional de La Plata-CONICET, La Plata, Buenos Aires, Argentina, 46 pp. 